\title{
Optimization of a Diamond Nitrogen Vacancy Centre Magnetometer for Sensing of Biological Signals
}

\author{
James L. Webb ${ }^{1 *}$, Luca Troise ${ }^{1}$, Nikolaj W. Hansen ${ }^{2}$, Jocelyn Achard ${ }^{3}$, Ovidiu Brinza ${ }^{3}$, \\ Robert Staacke ${ }^{4}$, Michael Kieschnick ${ }^{4}$, Jan Meijer ${ }^{4}$, Jean-François Perrier ${ }^{2}$, \\ Kirstine Berg-Sørensen ${ }^{5}$, Alexander Huck ${ }^{1}$ and Ulrik Lund Andersen ${ }^{1}$ \\ ${ }^{1}$ Department of Physics, Center for Macroscopic Quantum States (bigQ), Technical University of Denmark, Kongens Lyngby, \\ Denmark, ${ }^{2}$ Department of Neuroscience, Copenhagen University, Copenhagen, Denmark, ${ }^{3}$ Laboratoire des Sciences des \\ Procédés et des Matériaux, CNRS, Université Paris 13, Sorbonne Paris Cité, Paris, France, ${ }^{4}$ Division Applied Quantum \\ System, Felix Bloch Institute for Solid State Physics, University of Leipzig, Leipzig, Germany, ${ }^{5}$ Department of Health \\ Technology, Technical University of Denmark, Kongens Lyngby, Denmark
}

OPEN ACCESS

Edited by:

Carlo Manzo,

Universitat de Vic-Universitat Central

de Catalunya, Spain

Reviewed by:

Liam Terres Hall,

The University of Melbourne, Australia

Takeshi Ohshima,

National Institutes for Quantum and Radiological Science and Technology,

Japan

Anne Fabricant,

Helmholtz Institute Mainz, Germany

*Correspondence:

James L. Webb

jaluwe@fysik.dtu.dk

Specialty section: This article was submitted to

Biophysics,

a section of the journal

Frontiers in Physics

Received: 23 December 2019

Accepted: 28 August 2020

Published: 19 October 2020

Citation:

Webb JL, Troise L, Hansen NW, Achard J, Brinza O, Staacke R,

Kieschnick M, Meijer J, Perrier J-F,

Berg-Sørensen K, Huck A and Andersen UL (2020) Optimization of a

Diamond Nitrogen Vacancy Centre

Magnetometer for Sensing of

Biological Signals.

Front. Phys. 8:522536

doi: 10.3389/fphy.2020.522536
Sensing of signals from biological processes, such as action potential propagation in nerves, are essential for clinical diagnosis and basic understanding of physiology. Sensing can be performed electrically by placing sensor probes near or inside a living specimen or dissected tissue using well-established electrophysiology techniques. However, these electrical probe techniques have poor spatial resolution and cannot easily access tissue deep within a living subject, in particular within the brain. An alternative approach is to detect the magnetic field induced by the passage of the electrical signal, giving the equivalent readout without direct electrical contact. Such measurements are performed today using bulky and expensive superconducting sensors with poor spatial resolution. An alternative is to use nitrogen vacancy (NV) centers in diamond that promise biocompatibilty and high sensitivity without cryogenic cooling. In this work we present advances in biomagnetometry using NV centers, demonstrating magnetic field sensitivity of $\sim 100 \mathrm{pT} / \sqrt{\mathrm{Hz}}$ in the DC/low frequency range using a setup designed for biological measurements. Biocompatibility of the setup with a living sample (mouse brain slice) is studied and optimized, and we show work toward sensitivity improvements using a pulsed magnetometry scheme. In addition to the bulk magnetometry study, systematic artifacts in NV-ensemble widefield fluorescence imaging are investigated.

Keywords: diamond, biosensing and bioimaging, NV center in diamond, magnetic field, sensing

\section{INTRODUCTION}

Many biological processes generate electrical signals, for example synaptic transmission and muscular contraction. Such signals give key information on the functioning on biological systems, either for clinical diagnostic purposes (such as electrocardiography) or for fundamental understanding of processes and structure. Measuring these signals is typically performed using electrical probes carefully positioned in the desired region. This however poses difficulties if this region is not easily accessible, such as inside the brain, or where signals must be highly spatially resolved. An alternative approach is to measure the magnetic field produced when these signals propagate as electrical current. Existing techniques for this, such as magnetoencephalography (MEG) or magnetocardiography (MCG) are limited by reliance on superconducting quantum 
interference device (SQUID) systems [1]. These suffer a number of disadvantages including the need for an expensive magnetically shielded room and bulky cryogenic cooling, increasing the sample-sensor distance and resulting in weaker magnetic field at the sensor and poor spatial resolution.

A number of technologies are currently being researched in an effort to replace SQUIDs and overcome these difficulties, including microelectromechanical (MEMs) sensors [2], sensors based on magnetoresistive effects for magnetomyography [3] and in particular atomic (optically pumped) magnetometers for which cryogen-free MEG has recently been demonstrated [4]. Another alternative in the solid state is to sense magnetic fields using nitrogen vacancy (NV) centers in diamond [5]. Such defects, specifically the $\mathrm{NV}^{-}$center, have an electronic structure that results in magnetic field dependent variation in fluorescence output under illumination with resonant microwaves (MW) and $532 \mathrm{~nm}$ green laser light. Although as yet the sensitivity to magnetic field of NV sensors is worse than SQUIDs or atomic magnetometers, they have a number of advantages well-suited to biosensing. They operate under ambient conditions, from DC to $\mathrm{kHz}+$ bandwidth needed for ms-timescale biological signals and in close proximity (or even within [6]) cells or tissue, due to the excellent biocompatibility of diamond. The high density of such defects in the material also makes possible imaging of biological signals near the optical diffraction limit with micrometer scale resolution, in a way not possible by competing techniques. Finally, the high dynamic range of NV sensors means they can be operated without extensive magnetic shielding from ambient magnetic noise (e.g., from $50 / 60 \mathrm{~Hz}$ mains) and unlike atomic magnetometers, they do not require shielding from the Earth's magnetic field to achieve maximum sensitivity.

Figure 1A shows the simplified level structure of an $\mathrm{NV}^{-}$ defect in diamond used for such sensing. Electrons excited by a $532 \mathrm{~nm}$ pump laser can decay in two ways. The most likely, labeled (1) is directly in the triplet state between ground ${ }^{3} A_{2}$ and excited ${ }^{3} \mathrm{E}$ state. However, if microwaves (MW) are applied on resonance between the ground $\mathrm{m}_{s}=0{ }^{3} A_{2}$ state and its fine $\mathrm{m}_{s}$ $= \pm 1$ (and hyperfine) split levels $(2.8 \mathrm{GHz})$ followed by pump excitation, decay back to the ground state can occur through process (2), via singlet shelving states ${ }^{1} \mathrm{~A},{ }^{1} \mathrm{E}$ and non-radiative processes/weak infrared emission peaking at 1,042 $\mathrm{nm}$ [7]. This leads to a drop in fluorescence output on microwave resonance of up to $30 \%$ for a single NV, or $1-2 \%$ for a large NV ensemble [8]. This process is termed ODMR: optically detected magnetic resonance. The $\mathrm{m}_{s}= \pm 1$ states can be Zeeman split by a magnetic field, which shifts the resonance frequency, allowing detection of the field amplitude by monitoring fluorescence output. A $1 \mathrm{nT}$ field produces a shift of $28 \mathrm{~Hz}$ when the field is aligned along an $\mathrm{NV}$ axis and shifts down to a few $\mathrm{Hz}$ can be detected within 1 $s$ of acquisition time, corresponding to the picotesla level fields expected from biological signals.

Biosensing can cover bulk magnetometry, collecting all emitted fluorescence from a diamond and aiming for maximum overall sensitivity. It also covers widefield imaging magnetometry, aiming for realtime imaging of magnetic field at high resolution using a microscope objective and a camera. It can also cover scanning magnetometry using single or few NVs, via confocal microscopy, a scanning NV tip or nanodiamonds

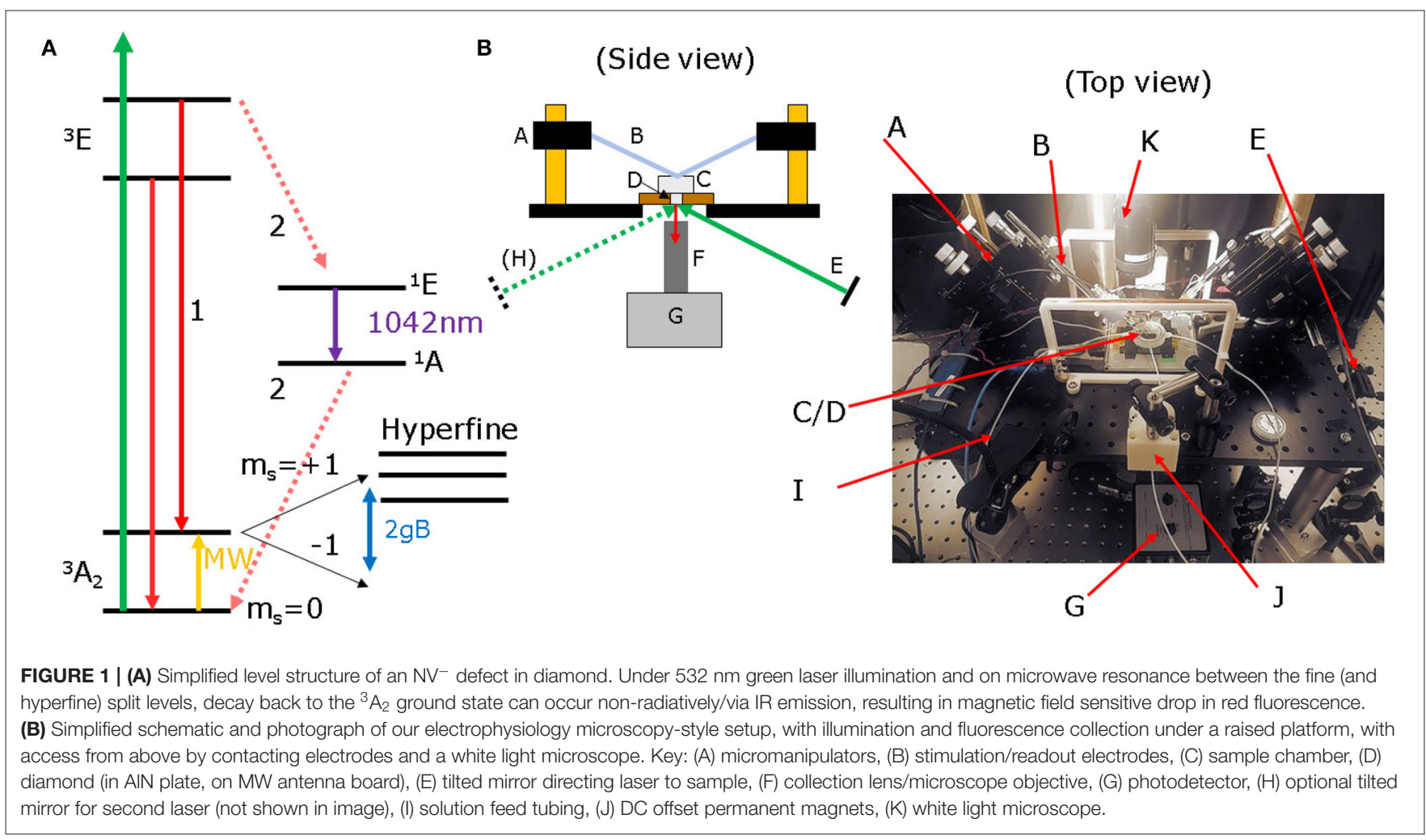


within a biological sample [9]. In this work we focus only the first two areas, bulk and widefield sensing. An excellent up to date summary of the field in general is given in the recent work by Barry et al. [10].

To date, bulk magnetometry biosensing has proved challenging. Barry et al. [11] demonstrated detection of single neuron action potentials from a marine worm, with excellent $15 \mathrm{pT} / \sqrt{\mathrm{Hz}}$ low frequency sensitivity. Few groups have yet to replicate this level of sensitivity, let alone on a biological specimen. We note a distinction between DC-low frequency sensitivity and the typically significantly better $\mathrm{AC}(>10 \mathrm{kHz})$ sensitivity achievable using pulsed dynamical decoupling techniques, where $10 \mathrm{pT} / \sqrt{\mathrm{Hz}}$ has been demonstrated [12-14]. These frequencies are however too high for detection of many biological signals in the sub-kHz range. The best reported low frequency/DC sensitivities are typically worse [15-17] with few-nT $/ \sqrt{H z}$. Vector magnetometry has also been demonstrated in the DC-low frequency range, important since magnetic fields from complex biosystems may not be easily directed along a single sensitive NV axis [18, 19].

For widefield imaging, a goal is to image the field from biological signals (such as neuron action potentials) [20, 21]. However, the pixel imaging sensitivity is on the order of 250 $\mathrm{nT}-2 \mu \mathrm{T} / \sqrt{\mathrm{Hz}}$, as yet insufficient for imaging pT-level fields [22]. Biosensing imaging work has therefore focused on samples which have a strong magnetic field due to the presence of ferromagnetic material [23-25]. Several works have studied magnetic microbeads, the tracking of which can have applications in biology [26]. The majority of work however has focused on imaging non-biological samples with far stronger magnetic fields, such as geological samples [27], vorticies in superconductors [28], and ferromagnetic nanowires [29].

In this work we present experimental measurements using bulk magnetometry and widefield sensing. We demonstrate a magnetometer setup with $100 \mathrm{pT} / \sqrt{\mathrm{Hz}}$ sensitivity which is designed and optimized for measurements of magnetic field from biological samples. We show the setup, in an inverted microscope geometry, is also well-suited for widefield imaging. We discuss the adaptations and considerations required for biological magnetometry and the potential limitations and pitfalls in terms of sample damage and imaging artifacts. Finally, we discuss the future prospects for these methods, with reference to our measurements using pulsed magnetometry.

\section{MATERIALS AND METHODS}

Our NV magnetometer setup is pictured in a schematic and photograph in Figure 1B. We use an inverted microscope geometry, where laser excitation and light collection is performed with the diamond held on a raised platform, above a microwave antenna board and below a custom-3D printed bath chamber for holding a biological sample (C and D, Figure 1), with the solution fed via capillary tubes (I) from a peristaltic pump. This configuration has developed from typical electrophysiology microscopy setups. The setup allows a test sample to be placed directly above the diamond, with easy access to the sample from above, allowing the entry of stimulating electrodes using 2-axis adjustable micromanipulators $(A, B)$ and a white light microscope (K) for positioning the electrodes and sample examination.

Fluorescence from the diamond can be collected from beneath the platform (F), using either a microscope objective or a condenser lens in a beam tube. Via a mirror, this is then directed to the photodetector $(\mathrm{G})$, placed below and infront of the raised platform. Using a beamsplitter, a reference beam is also supplied to the detector for noise rejection. Pump light is supplied from a tilted mirror (E) to the right of the setup at Brewster's angle for diamond $\left(67.5^{\circ}\right)$ to maximize coupling into the diamond (light can also be supplied from the left using a second tilted mirror $(\mathrm{H})$ from a second laser if required). Polarization of the pump light is controlled using a polarizing beamsplitter and half wave plate. Alternatively, both pump illumination and fluorescence collection can happen through the same lens using a dichroic mirror and optical filter to split the red/green components. This allows a focused beam to be supplied for imaging. In order to split the defect energy levels, optimize sensitivity in a single on-NV axis direction and obtain good contrast and sensitivity, a $\mathrm{mT}$ scale DC offset field is provided by two neodymium permanent magnets $(\mathrm{J})$ placed infront and behind the sample chamber along with field coils for applying test signals. To switch between bulk sensing and imaging, minimal simple adaptation is required: the condenser lens/beamtube $(\mathrm{F})$ is replaced with a microscope objective using the same screw fitting and the balanced photodetector $(\mathrm{G})$ replaced with a suitable camera.

\subsection{Diamond Material}

Careful selection of exposure dose, ${ }^{12} \mathrm{C}$ isotopic purification to remove the negative effects of ${ }^{13} \mathrm{C}$ spins $[30,31]$ and understanding the role of strain [32] are key to maximizing sensitivity by achieving narrow microwave resonance linewidths and high fluorescence contrast (change in output between microwaves on/off). In this work we use a diamond (grown at LSPM, Paris) with a $20 \mu \mathrm{m}$ CVD-grown ${ }^{12} \mathrm{C}$ purified layer, doped with 5 ppm nitrogen-14, carefully irradiated using $\mathrm{H}^{+}$ ions (at U. Leipzig) and subsequently annealed at $800^{\circ} \mathrm{C}$. Figure 2 shows ODMR for our ${ }^{12} \mathrm{C}$ purified diamond. The linewidth FWHM, proportional to the dephasing time $\mathrm{T}_{2}{ }^{*}[34]$, is on the order of $1 \mathrm{MHz}$, as compared $10-50 \mathrm{MHz}$ for high density and unpurified samples.

Peak sensitivity balances high NV number (high brightness) and ODMR contrast and linewidth. Brighter samples with higher NV density tend to have a lower contrast and broader linewidth and those with high contrast and narrow linewidths tend to be low in brightness. Further discussion of the sensitivity limitations, including the effects of laser line narrowing and microwave power broadening are given in the work by Dreau et al. [35]. Our diamond has a linewidth comparable to the better diamonds in literature (FWHM $\leq 1 \mathrm{MHz}$ ), contrast of $1-2 \%$ at maximum field sensitivity and total fluorescence collection of 5$6 \mathrm{~mW}$ at $2 \mathrm{~W}$ of pump power. Based on these values, a simple estimate based on realistic collection efficiencies gives a shot noise limited sensitivity to DC and low frequency fields on the order of 10-20 pT/ $\sqrt{H z}$. 


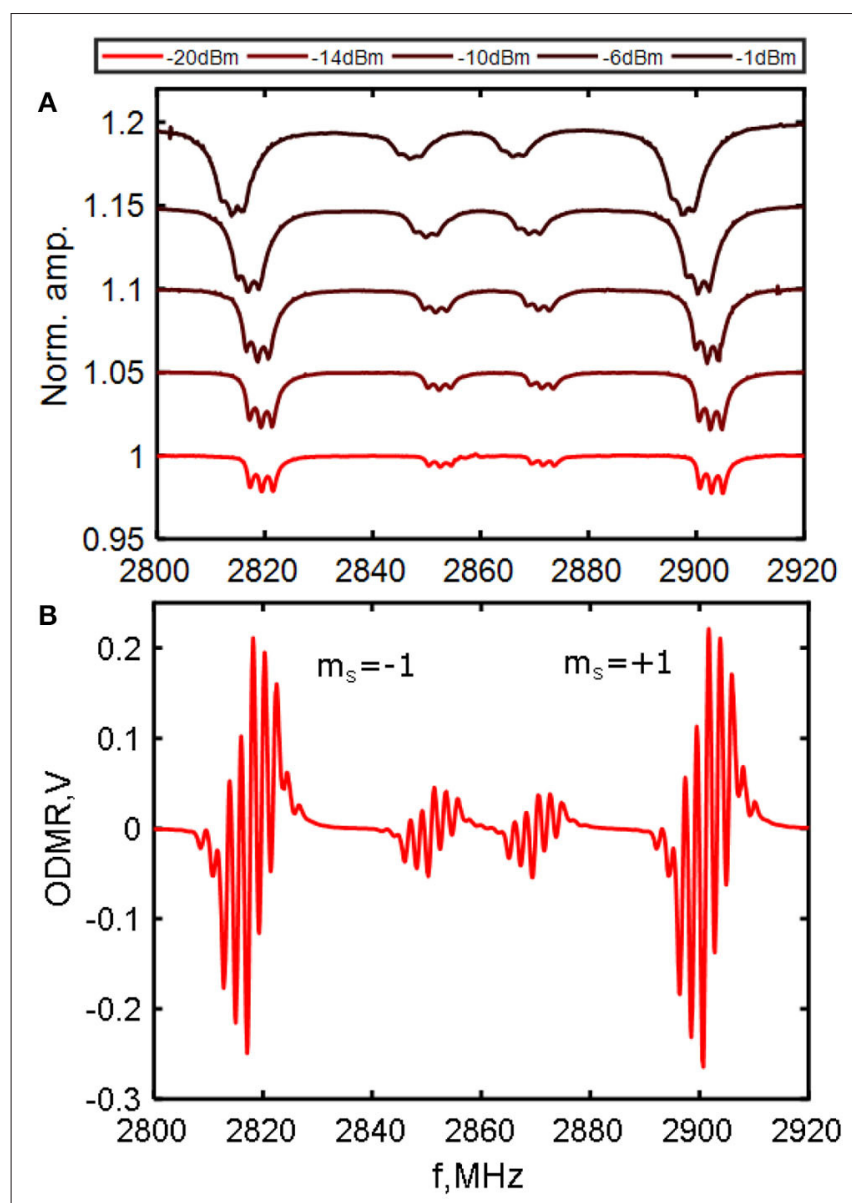

FIGURE 2 | ODMR from our diamond sample. The ${ }^{14} \mathrm{~N}$ diamond was ${ }^{12} \mathrm{C}$ enriched with $20 \mu \mathrm{m}$ of CVD overgrowth, then proton irradiated with $\mathrm{N}$ concentration $\approx 5 \mathrm{ppm}$. (A) Normalized change in DC photovoltage with $2 \mathrm{~W}$ pump power (max $6.5 \mathrm{~mW}$ fluorescence) at a range of microwave input powers, each offset by $0.05 . \mathrm{A} \approx 1.6 \mathrm{mT}$ offset field was applied with fine adjustment from field coils to overlap $2 \mathrm{NV}$ axes and boost contrast to a maximum of $5.1 \%$. Due to microwave broadening and resulting loss of the hyperfine features, actual peak sensitivity is reached at a lower $3.8 \%$ contrast. The downward frequency shift observed is due to heating by the microwave field. (B) ODMR at peak sensitivity using a $33 \mathrm{kHz}$ MW frequency modulation of $500 \mathrm{kHz}$ mixed with a 2.16 MHz hyperfine transition drive (see method details in the work by El-Ella et al. [33]). This boosts sensitivity to $100 \mathrm{pT} / \sqrt{\mathrm{Hz}}$. Linewidth FWHM is $\sim 1 \mathrm{MHz}$.

\subsection{Laser Coupling and Light Collection}

A factor critical to good performance in diamond $\mathrm{NV}$ magnetometry is coupling laser light in at Brewster's angle for diamond. This produces significant improvements in fluorescence generation by both coupling more pump light into the diamond and increasing internal reflection of the pump light, illuminating more NV centers [36]. This also has the additional benefit of minimizing damaging pump laser leakage into any biological sample. This configuration, used for all experiments in this work, is achieved in our setup using a tilted mirror, directing the light in from the right hand side of the setup. Using an extra dichroic mirror, our setup also allows pump light to be directed through a microscope objective, with light incident perpendicular to the sample. Due to the limited field of view, this however can lead to saturation in the ODMR amplitude and reduction in contrast and sensitivity at higher power. For imaging we therefore use a microscope objective just for fluorescence collection. For the Brewster's angle configuration, we rotated the polarization of the incident light using a half wave plate to ensure maximum transmission into the diamond.

Our setup was also designed to utilize more than one laser beam incident on the diamond at the correct angle, using an additional tilted mirror to the left hand side of the setup. Although laser light from two separate sources is not coherent, both beams can be sampled into the reference of a balanced photodetector in order to effectively reject the technical noise from both lasers simultaneously. This permits using two lower cost DPSS 1-2 W lasers in combination to achieve greater sensitivity, as compared to having to purchase a single, high power, high stability laser. For imaging, our collection efficiency was limited by the numerical aperture of the objective. For bulk magnetometry, we used an aspheric condenser lens (Thorlabs, antireflective coated) to maximize fluorescence collection.

\subsection{Detection and Sensing Limitations}

Sensing with an NV center ensemble is fundamentally limited by shot noise from the strong fluorescence background originating from transition directly from excited ${ }^{3} \mathrm{E}$ to ground ${ }^{3} A_{2}$ triplet state and the low 1-3\% change in fluorescence level on microwave resonance. This is the difficult task of detection of a small dip in brightness on a large bright background with a consequently high shot noise level. This shot noise limited regime means sensitivity improves with the square root of the number of NV centers (larger ensemble) and by high pump laser power (up to the order of Watts). In order to address the $\mathrm{NV}^{-}$defects and reach these power levels, we used a $532 \mathrm{~nm}$ DPSS laser (Coherent Verdi) of maximum power output $2 \mathrm{~W}$. We note that the majority of the fluorescence produced is trapped within the diamond by its high refractive index.

In practice, reaching this shot noise limited regime is difficult due to laser technical noise and electrical readout noise. In our setup, we rejected laser technical noise using a balanced photodetector (Newport Nirvana) or by sampling the input laser beam and digitally subtracting it from the fluorescence signal [19]. The majority of our electrical readout noise originates primarily from the transimpedance amplifiers within the detector and the choice of photodiode. For the balanced detector, we used the inbuilt photodiodes, with bandwidth limited by the electric balance feedback circuit to $100 \mathrm{kHz}$. For the alternative sampling and subtraction method, we chose a photodetector with a larger area photodiode and higher capacitance (Thorlabs DET100) to ensure maximum fluorescence detection, at the cost of higher noise. Detection bandwidth was $10 \mathrm{MHz}$, more than sufficient for $\mathrm{kHz}$ signal detection.

For the majority of this work sensing was performed by supplying microwaves frequency modulated at $33 \mathrm{kHz}$ with 500 $\mathrm{kHz}$ modulation width. This modulation could then be detected in the collected fluorescence from the diamond and the ODMR spectrum recovered by sweeping microwave frequency and 
performing detection using a lock-in amplifier. Once the ODMR spectrum had been obtained, a fixed microwave frequency was used corresponding to the point of maximum slope and thus giving the maximum response to any magnetic field induced change in fluorescence output. We used a three-frequency drive method as outlined in El-Ella et al. [33]. Measurements were performed using a continuous wave method with constant microwave and laser power.

In the section Pulsed Measurement of this work, short microwave and laser pulses were instead used for sensing, measuring the change in output on the readout laser pulse with and without a prior microwave pulse. Pulses were generated from a TTL pulse generator (Spincore Pulseblaster) and fed to fast RF switches (Minicircuits) and an acousto-optic modulator (Isomet). The objective of the pulsed scheme was to reduce microwave power broadening effects and enhance ODMR contrast $[5,8]$.

\subsection{Measurement Environment}

In order to maintain optimum sensitivity, it is necessary to well control the environment in proximity to the diamond. This is difficult to achieve in practice due to the need to hold biological samples in solution that can vary in volume or composition over time, due to movement (particularly muscle tissue) and due to changes in temperature, all of which can shift the optimum microwave frequency and power required to maximize magnetometer sensitivity.

The efficiency of microwave coupling to the diamond can be considerably affected by having a water or other solution in close proximity. This is particularly critical for antennas with a narrow resonance, such as wire loops [37]. To counter this, we fabricated a custom-designed broadband antenna on a РСВ board to be placed adjacent to the diamond. Using this antenna, we could reoptimize MW power (often by several orders of magnitude) after a stable carbogenated solution level sufficient to hold the specimen within was reached. Typically a depth of $0.5-1 \mathrm{~cm}$ was used, defined by the need to fully cover and contain within the solution a biological sample, such as a tissue slice or a mouse muscle. In order to maintain sensitivity, it was critical to keep a constant solution level and constant flow (provided by a peristaltic pump), avoiding shifts in the MW resonance. Stabilization of the level could be passively achieved by careful custom chamber design for the pumping rate required, by manually constricting a section of tubing to reduce inflow or by controlling the rate of inflow or outflow using needle valves in the feed and return piping. These could either be manually operated or controlled from a level sensor on the chamber. If necessary to fully inhibit sample movement, inhibitor chemicals as butanedione monoxime (BDM) (dissected tissue) or local anesthetic (living specimen) could be introduced to the solution feed and pumped to the sample.

Another factor was gradual thermal drift, caused by microwave or (as previously discussed) laser heating. Thermal drift could, over the course of minutes, push the optimum microwave resonance frequency away from the setpoint frequency, reducing sensitivity. To correct for this, we designed automated software to perform an ODMR sweep every few measurements, with the computer automatically determining the point of maximum field sensitivity and maintaining the microwave frequency at this point. This could alternatively be performed continuously by keeping the frequency at a point that maximized the strength of a low frequency $(111 \mathrm{~Hz})$ signal applied to test coils aligned with the field sensitive axis.

\subsection{Sample-Diamond Separation}

Since magnetic field strength drops as the inverse of distance (current carrying wire approximation) or the cube of the distance (magnetic dipole approximation), only a small difference in separation between diamond and biological sample (order of $\mu \mathrm{m}$ [22]) can make a significant difference in signal detection strength. This poses particular challenges for biological systems, where the tissue generating the signal may unavoidably be many millimeters from the sample (such as within muscle or under bone) or cannot be directly in thermal contact with a diamond heated by a laser beam. In our setup, we used a reflector/insulator layer above the diamond (aluminum foil/Kapton tape) to keep the sample as close to the diamond as possible while minimizing heat transport from diamond to sample. We also found that samples could float from the surface away from the diamond. Using a Pt and nylon harp (standard equipment for electrophysiology) helped stop this without disrupting the DC offset magnetic field. We also used Ti hooks and electrodes to hold the sample onto the diamond, with care taken not to damage the sample.

\subsection{Stimulating Electrode Type and Design}

In order to stimulate a biological response, our setup was configured to use standard electrophysiology probes attached to micromanipulators, which could supply current pulses to the sample. In electrophysiology experiments, only the electrical performance of the test electrodes is important and no offset magnetic field is used. This means many types of electrical stimulation electrodes that can be purchased contain magnetizable steel, often in the outer sheath of concentric-type electrodes. Such electrodes become magnetized in the DC offset field, which disrupts the field close to the diamond, significantly reducing magnetometer performance. To avoid this problem we developed an alternative, which was to use custom-made electrodes using $\mathrm{Pt} / \mathrm{Ir}$ wire with a glass or plastic outer sheath. We found similar electrodes could be made from biocompatible, non-ferrous $\mathrm{Ti}$ or $\mathrm{W}$ wire $(\mathrm{Cu}$ was not biocompatible unless coated with $\mathrm{Au}$ or $\mathrm{Ag} / \mathrm{AgCl})$.

\subsection{Averaging}

Due to the weakness of biological signals, it is necessary to repeat the signal $\mathrm{N}$ times and then average, with improvement in sensitivity proportional to $1 / \sqrt{N}$. This however places considerable demands on a biological sample, for example due to fatigue in a muscle, due to damage induced in the tissue by repeated stimulation or due to thermal degradation. We found that a bath of carbogenated (5\% carbon dioxide, 95\% oxygen) solution allowed the sample to survive for many hours, permitting many thousands of stimulations and averages. 


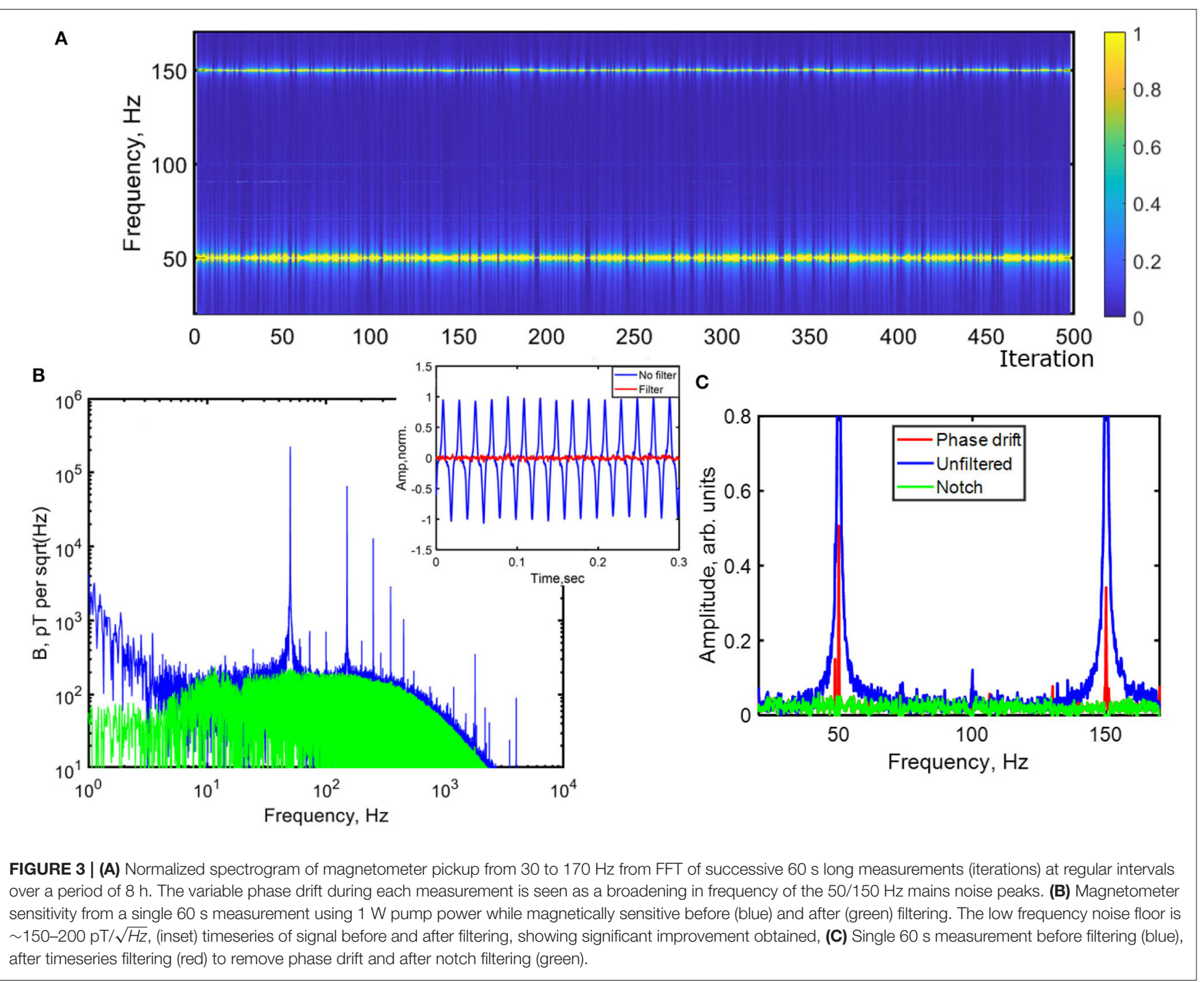

\section{RESULTS}

\subsection{Magnetic Noise}

In any practical laboratory or clinical setting, there will be substantial magnetic field generation from mains transformers in equipment and in building wiring. This occurs primarily at the fundamental mains frequency (European $50 \mathrm{~Hz}$ or North American $60 \mathrm{~Hz})$ and the $3 \mathrm{rd}$ harmonic $(150$ or $180 \mathrm{~Hz}$ ) arising from magnetic hysteresis in the transformer, but also at higher harmonics and at multiples of three phase frequencies. Such low frequency fields are difficult to shield against, requiring full mu-metal enclosure, impractical for processing of biological samples, or extremely costly fully shielded rooms into which feeding piping or cabling is equally hard. Active cancellation, by using field coils to generate a counter field to the noise, can reduce it but this is hard to achieve for multi-axis sensing at a small $1-2 \mathrm{~mm}$ diamond, at $\mathrm{kHz}$ bandwidth and operating at sub-nT/ $\sqrt{\mathrm{Hz}}$ sensitivity. The majority of commercially available systems cannot achieve these levels of field cancellation.
The alternative we present here is for the magnetically sensitive signal from the diamond magnetometer to be processed and filtered by Fast Fourier Transform (FFT) methods. This requires not only effective filtering but minimal filtering, since many biological signals have frequency components in the 10$500 \mathrm{~Hz}$ range and any strong filtering here will distort or remove the desired signal entirely. Such a filter must adapt to any drift in mains frequency and be able to capture any transient noise (such as from a fan or pump turning on and off). Further, it must counter phase drift in the mains supply, which acts to spread the mains noise across a wider frequency range.

In Figure 3 we demonstrate how effective this can be. Magnetometer data was collected for $60 \mathrm{~s}$ in order to give good frequency discrimination. Low frequency and DC drift arising from laser power fluctuations is first removed by a highpass filter at $10 \mathrm{~Hz}$. Next, a $50 \mathrm{~Hz}$ zero phase pure sine wave is correlated with a readout of the fundamental mains frequency obtained from tapping input to a transformer, in order to obtain a time series of the phase drift over the measurement period. A slight 
constant phase shift can be added to this signal to account for any phase shift due to inductive effects, then normalized and subtracted from the magnetic readout. This process could be repeated several times to remove multiple (additive) sources of noise with different phase. It can then be repeated at 150, 250 $\mathrm{Hz}$, or other strong harmonics of mains. Consequently, the phase drift broadening is removed in the frequency domain. Narrow notch or bandstop filters can then be used, centered on the noise frequencies, to remove any remaining noise components (Figure 3C). Any high frequency RF noise could then be removed by a final lowpass filter at $5-10 \mathrm{kHz}$. The overall effect in the data is a reduction in noise by orders of magnitude, down to the electrical and optical noise floors (inset, Figure 3B).

The ability to remove strong background magnetic noise components is key to allowing diamond NV sensing to be used in an ordinary lab or clinical environment without extensive and costly magnetic shielding for applications in research and medical diagnosis (e.g., magnetocardiography). This work complements our recent work on a flexible handheld magnetometer, containing the diamond, focusing optics and electronic components [38].

\subsection{Laser Heating}

In order to optimize sensitivity in NV center experiments limited by fluorescence shot noise, high laser powers of the order of several Watts are required. This presents a challenge of focusing a high power visible laser beam onto a mm-size diamond while remaining within the $\leq 37^{\circ} \mathrm{C}$ range from most biological processes. We found that sudden changes in temperature, such as from fast ramping of the laser to high power, would damage biological samples. Heat must be dissipated from the diamond while minimizing the distance between diamond and sample, to maximize magnetic field strength at the diamond. Samples often cannot be placed directly on a metal heatsink, as this would short out any conduction paths and potentially interfere with the microwave or DC magnetic offset fields.

Figure 4 shows the effect of slow ramping the laser power on the electrical readout on the local field potential evoked in the CA1 region of the hippocampus in a slice preparation of a mouse brain. Placing the sample directly onto the diamond surface, heated by the pump laser, was found to cause rapid damage or death to the slice. By using a metal foil layer as heatsink and to reflect stray laser light back into the diamond and thinner Kapton tape as electrical insulation, it was possible to ramp the laser slowly to high power while keeping the sample alive, with the signal strength slightly improving through gradual heating to a more optimal temperature for biological processes $\left(35-37^{\circ} \mathrm{C}\right)$. We observed no signal for direct slice transfer to a diamond preheated by the laser at 1 or $2 \mathrm{~W}$, attributable to thermal shock on placing it on the warm diamond. Ideally, the foil/Kapton layers could be replaced with thin glass or polymer (to be more robust against pressure from above) coated with $\mathrm{Al}$ to form a mirror. It should be noted that standard commercial glass coverslips are too thick for this purpose (type \#0 are 85-105 $\mu \mathrm{m}$ thick).

One additional way of achieving good thermal dissipation is to have the diamond in contact with a SiC wafer [11]. Another method we utilize in this work was to use plates of aluminum

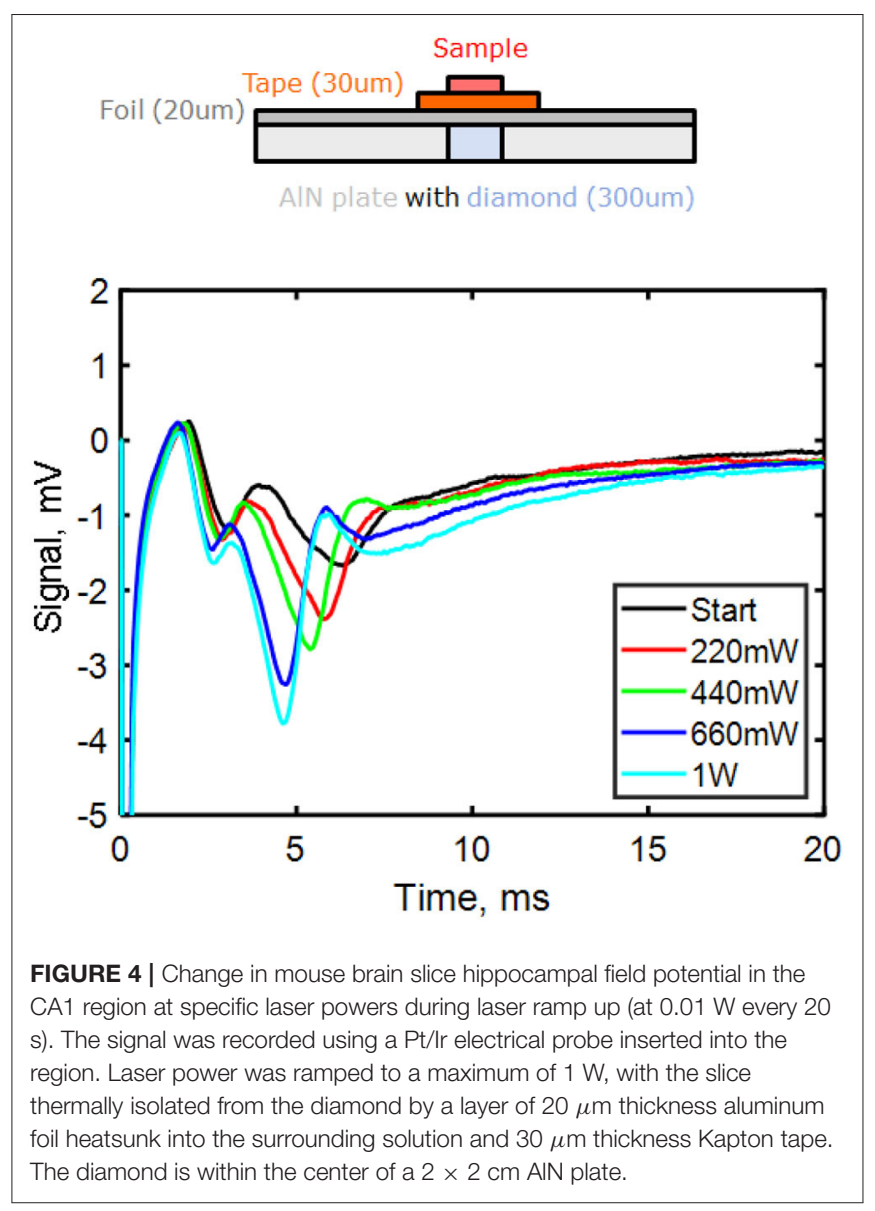

nitride (AlN) with a precision laser cut hole for the diamond. AlN is cheaper to purchase, easier to machine and has a good thermal conductivity while being an electrical insulator. This allowed heat to be dissipated out from the side facets of the diamond. We note from our previous work that thermal effects on the magnetometry can be canceled by driving two $\mathrm{m}_{s}= \pm 1$ hyperfine transitions, although at a factor $2 \times$ cost in sensitivity [39].

Although we present electrophysiology measurements from the hippocampal slice here, we were unable to observe these signals in the magnetometer readout. Our previous estimates (detailed methodology in Karadas et al. [22]) had indicated that reaching $\mathrm{nT} / \sqrt{\mathrm{Hz}}$ sensitivity along with signal filtering and averaging would be sufficient to resolve signals from a brain slice. We speculate that this failure was due to magnetic field cancellation at the diamond. We consider that this occurs due to current propagation in other directions in the slice than the desired direction we impose using the permanent magnet DC bias field to maximize the magnetic field along the most sensitive $\mathrm{NV}$ axis. We found it extremely difficult to position the slice (in a solution bath) correctly to direct the current in the hippocampus. Higher spatial resolution and/or vector magnetometry is required in order to probe the cause of the absence of magnetic signal. In our setup this could not be achieved without reducing ensemble size, fluorescence collection and excessively reducing sensitivity. 

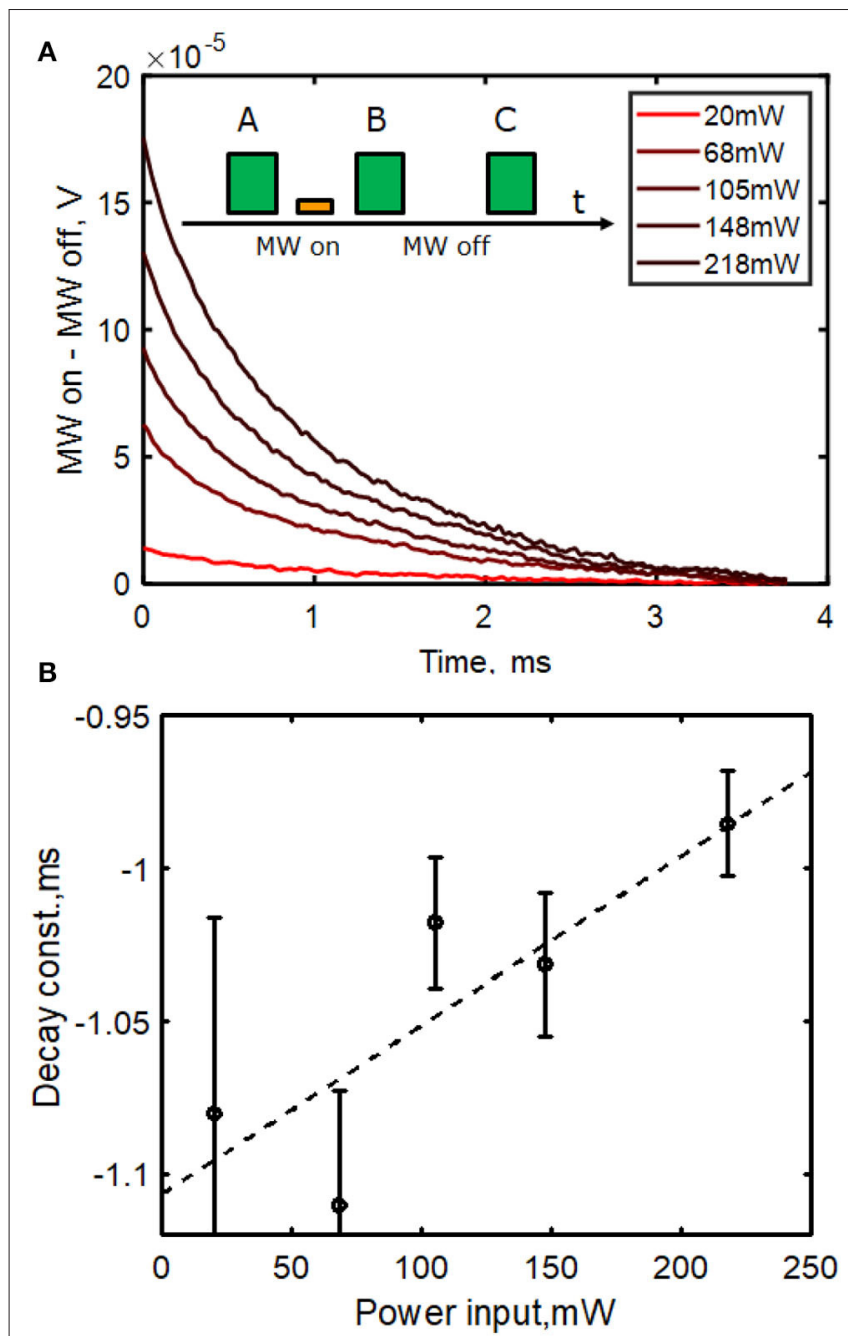

FIGURE 5 | (A) Difference in fluorescence vs. readout pulse time (B,C). The system is initialized with a laser pulse A. Before B a $680 \mathrm{~ns} \pi$ microwave pulse is applied (MW on) and before C, no MW pulse is applied (MW off). The difference in photodetector output voltage during these two pulses as a function of time was measured and the difference is plotted in the figure (MW on-MW off, V). Data is taken at a range of pump laser powers from a Gaussian beam of waist $200 \mu \mathrm{m}$ incident at Brewster's angle. (B) Fitting to an exponential with error estimates based on readout noise. Typical decay length was $1 \mathrm{~ms}$, reducing with higher pump power. This long readout time restricts field sensing bandwidth to 250 or $83 \mathrm{~Hz}$ for a 3 pulse, common mode noise rejection scheme. Linear fit (dashed) $y=m x+c$ coefficients are $m=5.5 \times$ $10^{-4} \mathrm{~ms} / \mathrm{mW}$ and $\mathrm{c}=-1.1 \mathrm{~ms}$.

Although widefield imaging microscopy cannot resolve pT-level signals at the present time, this or scanning either the sample or the diamond on a probe over the biological sample may prove a method to overcome this issue in the future.

\subsection{Pulsed Measurement}

Substantial effort in recent years has gone into developing pulsed laser/microwave schemes for field detection and imaging. These have been very successful in improving sensitivity to high frequency AC fields $(>10 \mathrm{kHz})$ via dynamical decoupling methods, rather than in the limit of low frequency and DC signals. A significant problem for DC sensing is the time required to read a large ensemble of NV centers required to achieve a high level of sensitivity. This can limit the measurement bandwidth to below that required for many biological sensing applications, particularly if laser power must be limited in order to protect the biological sample from damage.

Here we measured the ensemble readout time for our diamond. We used a long (100 ms) initialization laser pulse (A) to ensure that as many NVs as possible were initialized in the $\mathrm{m}_{\mathcal{s}}$ $=0$ ground state. We then sent a microwave pulse (on resonance at $2.91 \mathrm{GHz}$ ) of variable length $\tau$ (MW on) This was followed by a second laser readout pulse (B), followed by a repeat of this sequence without the microwave pulse (MW off), finishing with a second readout pulse (C) (see schematic, Figure 5A). We measured the difference in fluorescence output between the readout pulse with $\mathrm{MW}$ on (A) and MW off (B) as a function of time, as would be necessary in a measurement scheme, such as Ramsey interferometry. We varied the length of the microwave pulse $\tau$ to maximize this difference, which was found at $\tau=680$ ns. For a single NV, this would correspond to a $\pi$-pulse. For our large ensemble, this corresponds to an approximate $\pi$-pulse for the most number (far from all) NVs. Figure 5 shows the result of these measurements, measuring the difference in fluorescence (detected photovoltage) as a function of readout laser pulse time and averaging over 1,000 repetitions of the pulse sequence. We find a readout (reinitialization) time with a decay time on the order of $1 \mathrm{~ms}$, fitting using a single exponential function. We estimate the error bars on the exponential fit in Figure 5B) based on the maximum deviation in fit parameter produced by the peak to peak readout noise on the photodetector voltage, which decreased with higher laser power (more fluorescence signal). Within error bounds, the decay time could be decreased by 200-300 $\mu$ s by increasing laser power from 20 to $218 \mathrm{~mW}$, the maximum possible using this measurement configuration due to power limitations imposed by our acousto-optic modulator. We estimate the laser power intensity at the diamond to be 1.5 $\mathrm{kW} / \mathrm{cm}^{2}$. Assuming a linear extrapolation to the higher power density used, our readout time is comparable to the $150 \mu$ s in the work by Wolf et al. [13]. This is likely due to the reason given in their work, related to Gaussian spread of pump laser power, but also due to variations in strain and local field across the diamond and NV ensemble.

We note that for a large ensemble, a constant amplitude and phase microwave pulse will not correctly address all NVs, either as a $\pi$-pulse in this example or as a $\pi / 2$-pulse for a sensing scheme based on Ramsey interferometry [10]. A prospect to overcome this and enhance sensitivity in the pulsed regime may be microwave pulse shaping, using an arbitrary waveform generator and optimal control methods to boost readout fidelity [40].

\subsection{Widefield Imaging}

The camera limitations of NV center widefield fluorescence imaging are discussed in detail in our recent work [39]. A key problem with NV ensemble widefield imaging is bit depth. The majority of digital cameras in general and used for microscopy 


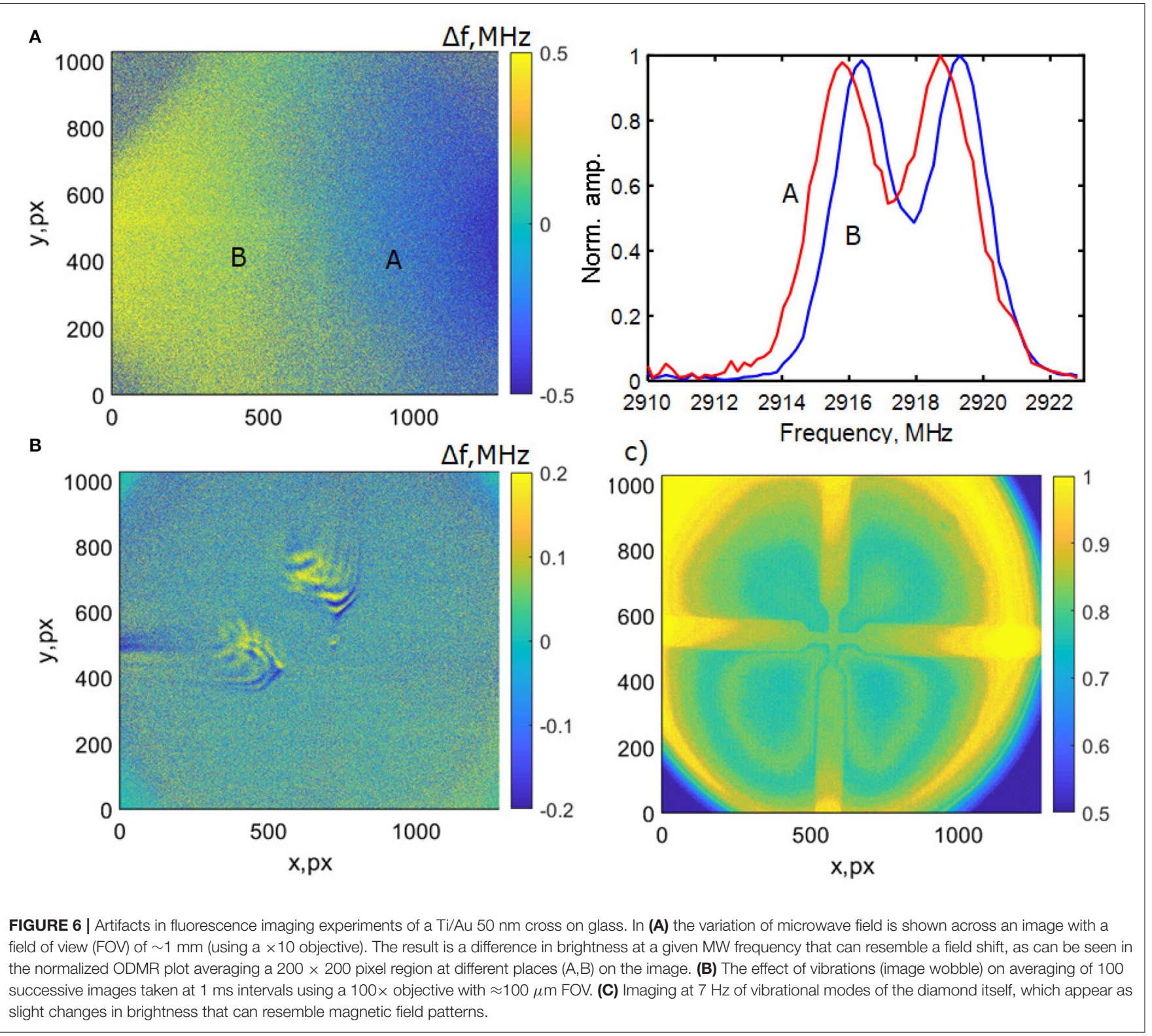

work in only 8 or 10 bit depth (since the human eye cannot distinguish a greater color or monochrome palette). With a large background fluorescence brightness, this can result in the on MW resonance contrast being spread across too few digitization levels. We therefore use more specialized 12 and 16 bit cameras (Imaging Development Systems $\mathrm{GmbH}$ ) in order to avoid artifacts associated with low bit depth. An ideal camera would be one with an initial analog black level correction stage, that can spread the contrast across the full digitization range.

Such a high level of background brightness means shot noise will significantly exceed readout noise or dark noise on each pixel. Based on ODMR measurements and from technical specifications, we can estimate the best sensitivity possible. Assuming a detection area of $5 \times 5 \mathrm{~mm}^{2}$ with $3.5 \mu \mathrm{m}^{2}$ pixel size ( $\sim 2$ Mpixels) and a diamond with a ODMR linewidth of $1 \mathrm{MHz}$, contrast of $2 \%$ and collecting a fluorescence output of $\approx 6 \mathrm{~mW}$, we calculate the best per pixel shot noise limited sensitivity of an imaging system to be $\sim 50 \mathrm{nT} / \sqrt{\mathrm{Hz}}$. This presently restricts imaging of biological signals to specific, low bandwidth cases rather than imaging of $\mathrm{pT}$-level signals, such as those from neural activity.

A key issue we address in this work is imaging artifacts that can easily resemble magnetic fields. From our measurements, we demonstrate a number of these in Figure 6. Variation in microwave power and resonance frequency across the image width can be more significant than the shift resulting from local changes in magnetic field (Figure 6A), which can in turn be mistaken for localized magnetic field imaging. Jitter or wobble in repeated averaged images can produce patterns that appear to be localized to electrodes or areas where a field is 
expected (Figure 6B). In addition, low frequency vibration of the diamond itself in the typical frequency range of biological signals can be observed in a magnetic-field like pattern of fluorescence brightness change (Figure 6C). Finally, magnetic field-resembling diffraction patterns can arise from imperfect interfaces and air or solution gaps between diamond and sample.

\section{DISCUSSION}

Improved sensitivity is key for biosensing, since this would allow greater diamond-sample separation while still being able to detect the signal, would allow more comprehensive measurement and filtering of background noise and would permit biological magnetic field imaging of even weaker signals (i.e., sub-pT to fT level for MEG). Substantial work in the field has focused on using pulse sequences to improve sensitivity [10]. This method appears challenging for DC/low frequency sensing, due to the bandwidth limitations of slow readout times for large ensembles, although microwave pulse shaping and optimal control techniques common in nuclear magnetic or electron spin resonance experiments (NMR/ESR) may help here. A number of novel sensing techniques are currently being pursued which are suitable for sensing in this low frequency regime. This includes experiments using an optical cavity and infrared or green absorption [41, 42] or the very recent work by Fescenko et al. [43] promising sub-pT/ $\sqrt{\mathrm{Hz}}$ sensitivity using flux concentrators. Novel techniques at an early stage, such as laser threshold magnetometry [44, 45] also appear promising. Such laser threshold experiments can potentially work with other materials beyond diamond, avoiding the problem of the large shot noise background from spin triplet fluorescence emission. Another possibility we suggest here is that pump light absorption may be exploited using interferometry techniques, such as by placing the diamond with the sample in one arm of a Mach-Zehnder configuration and detecting magnetic field through the effect of the field-dependent absorption of the diamond on the interference pattern generated at the output. This requires minimizing reflective losses and good stable transmission through the diamond in order to be realized.

It should be noted that many of these schemes pose problems for positioning the biological sample near the diamond yet out of the beam path. However, with sufficient sensitivity, the stand-off distance between the sample and the diamond can be increased (as is already necessary and possible for SQUID and atomic magnetometers), allowing it to be displaced laterally from the diamond while maintaining an orientation that directs the magnetic field along a sensitive NV axis. Many of these novel schemes are at an early stage and require further experiments to test their viability before applications to biosensing can be considered.

In this work, we have demonstrated methods that avoid damage to biological samples. Further work is however required in terms of improving dissipation of heat away from the diamond and away from the sample. This is challenging since any heatsink must be thin to minimize sample-diamond separation and also electrically insulating. We note that there has been important parallel effort in constructing integrated miniaturized sensors [46-48]. Although their reported sensitivity is several orders of magnitude worse, it is possible that by using techniques from semiconductor fabrication (particularly efficient heatsinking) that these devices may have a significant role to play in future NV biosensing.

Finally, it is hoped that advances in diamond material processing may drive future development. In particular, improvements in CVD growth, nitrogen levels and conversion to $\mathrm{NV}^{-}$centers, optimization of irradiation and dealing with material strain. Patterning of the diamond may allow a greater fraction of the fluorescence to escape, boosting efficiency [49].

\section{CONCLUSION}

Sensing of biological signals via their magnetic fields using diamond NV centers provides a potential route to better measure and understand them, whether in an electrophysiologystyle microscopy configuration or from MEG-style sensing of inaccessible tissues in a living subject. Previous work has shown the technique to be biocompatible and widely applicable to a number of different systems, in both a bulk sensing and imaging configuration. There are, however, challenges in realizing such measurements, both in general from material limitations and the need to filter out unwanted noise, to specific demands unique to biosensing, such as maintaining temperature stability. In the coming years, further advances in sensitivity are required in order to realize ambitious sensing goals. A number of promising ideas are currently under development, particularly using pulsed schemes and in improving diamond material quality.

\section{DATA AVAILABILITY STATEMENT}

The datasets generated for this study are available on request to the corresponding author.

\section{ETHICS STATEMENT}

The animal study was reviewed and approved by AH, UA, and J-FP. Research was conducted according to applicable national laws and guidelines and local rules at DTU/KU.

\section{AUTHOR CONTRIBUTIONS}

JLW, NH, and LT performed the experiments for which data is shown as figures in this work. JLW wrote the manuscript with input from AH, KB-S, UA, J-FP, and NH. All authors contributed to the article and approved the submitted version.

\section{FUNDING}

The work presented here was funded by the Novo Nordisk Foundation through the synergy grant bioQ (NNF17OC0028086) and the Center for Macroscopic Quantum States-bigQ (bigQDNRF142) funded by the Danish National Research Foundation (DNRF). 


\section{ACKNOWLEDGMENTS}

We acknowledge the assistance of Kristian Hagsted Rasmussen for fabrication and diamond processing and Axel Thielscher,
Mursel Karadas (current and former DTU Heath Tech), and Adam Wojciechowski (former DTU Physics, currently Jagiellonian University, Krakow) for contributions and prior experimental and theoretical modeling work.

\section{REFERENCES}

1. Körber R, Storm JH, Seton H, Mäkelä JP, Paetau R, Parkkonen L, et al. SQUIDs in biomagnetism: a roadmap towards improved healthcare. Supercond Sci Technol. (2016) 29:113001. doi: 10.1088/0953-2048/29/11/113001

2. Li M, Matyushov A, Dong C, Chen H, Lin H, Nan T, et al. Ultra-sensitive NEMS magnetoelectric sensor for picotesla DC magnetic field detection. Appl Phys Lett. (2017) 110:143510. doi: 10.1063/1.4979694

3. Zuo S, Heidari H, Farina D, Nazarpour K. Miniaturized magnetic sensors for implantable magnetomyography. Adv Mater Technol. (2020) 5:2000185. doi: 10.1002/admt.202000185

4. Boto E, Holmes N, Leggett J, Roberts G, Shah V, Meyer SS, et al. Moving magnetoencephalography towards real-world applications with a wearable system. Nature. (2018) 555:657-61. doi: 10.1038/nature26147

5. Taylor JM, Cappellaro P, Childress L, Jiang L, Budker D, Hemmer PR, et al. High-sensitivity diamond magnetometer with nanoscale resolution. Nat Phys. (2008) 4:810-6. doi: 10.1038/nphys1075

6. McGuinness LP, Yan Y, Stacey A, Simpson DA, Hall LT, Maclaurin D, et al. Quantum measurement and orientation tracking of fluorescent nanodiamonds inside living cells. Nat Nanotechnol. (2011) 6:358-63. doi: 10.1038/nnano.2011.64

7. Acosta VM, Jarmola A, Bauch E, Budker D. Optical properties of the nitrogen-vacancy singlet levels in diamond. Phys Rev B. (2010) 82:201202. doi: $10.1103 /$ physrevb.82.201202

8. Rondin L, Tetienne JP, Hingant T, Roch JF, Maletinsky P, Jacques V. Magnetometry with nitrogen-vacancy defects in diamond. Rep Prog Phys. (2014) 77:056503. doi: 10.1088/0034-4885/77/5/056503

9. Schirhagl R, Chang K, Loretz M, Degen CL. Nitrogen-vacancy centers in diamond: nanoscale sensors for physics and biology. Annu Rev Phys Chem. (2014) 65:83-105. doi: 10.1146/annurev-physchem-040513-103659

10. Barry JF, Schloss JM, Bauch E, Turner MJ, Hart CA, Pham LM, et al. Sensitivity optimization for NV-diamond magnetometry. Rev Mod Phys. (2020) 92:015004. doi: 10.1103/RevModPhys.92.015004

11. Barry JF, Turner MJ, Schloss JM, Glenn DR, Song Y, Lukin MD, et al. Optical magnetic detection of single-neuron action potentials using quantum defects in diamond. Proc Natl Acad Sci USA. (2016) 113:14133-8. doi: 10.1073/pnas.1601513113

12. Hall LT, Hill CD, Cole JH, Hollenberg LCL. Ultrasensitive diamond magnetometry using optimal dynamic decoupling. Phys Rev B. (2010) 82:045208. doi: 10.1103/physrevb.82.045208

13. Wolf T, Neumann P, Nakamura K, Sumiya H, Ohshima T, Isoya J, et al. Subpicotesla diamond magnetometry. Phys Rev X. (2015) 5:041001. doi: 10.1103/physrevx.5.041001

14. Masuyama $\mathrm{Y}$, Mizuno K, Ozawa H, Ishiwata H, Hatano $\mathrm{Y}$, Ohshima T, et al. Extending coherence time of macro-scale diamond magnetometer by dynamical decoupling with coplanar waveguide resonator. Rev Sci Instrum. (2018) 89:125007. doi: 10.1063/1.5047078

15. Zheng $\mathrm{H}, \mathrm{Xu}$ J, Iwata GZ, Lenz T, Michl J, Yavkin B, et al. Zero-field magnetometry based on nitrogen-vacancy ensembles in diamond. Phys Rev Appl. (2019) 11:064068. doi: 10.1103/physrevapplied.11.064068

16. Zheng H, Sun Z, Chatzidrosos G, Zhang C, Nakamura K, Sumiya H, et al. Microwave-free vector magnetometry with nitrogen-vacancy centers along a single axis in diamond. Phys Rev Appl. (2020) 13:044023. doi: 10.1103/PhysRevApplied.13.044023

17. Clevenson H, Trusheim ME, Teale C, Schröder T, Braje D, Englund D. Broadband magnetometry and temperature sensing with a light-trapping diamond waveguide. Nat Phys. (2015) 11:393-7. doi: 10.1038/nphys3291

18. Zhao B, Guo H, Zhao R, Du F, Li Z, Wang L, et al. High-sensitivity threeaxis vector magnetometry using electron spin ensembles in single-crystal diamond. IEEE Magn Lett. (2019) 10:1-4. doi: 10.1109/lmag.2019.2891616

19. Schloss JM, Barry JF, Turner MJ, Walsworth RL. Simultaneous broadband vector magnetometry using solid-state spins. Phys Rev Appl. (2018) 10:034044. doi: 10.1103/physrevapplied.10.034044

20. Hall LT, Beart GCG, Thomas EA, Simpson DA, McGuinness LP, Cole JH, et al. High spatial and temporal resolution wide-field imaging of neuron activity using quantum NV-diamond. Sci Rep. (2012) 2:401. doi: 10.1038/srep 00401

21. Hall LT, Simpson DA, Hollenberg LCL. Nanoscale sensing and imaging in biology using the nitrogen-vacancy center in diamond. MRS Bull. (2013) 38:162-7. doi: $10.1557 / \mathrm{mrs} .2013 .24$

22. Karadas M, Wojciechowski AM, Huck A, Dalby NO, Andersen UL, Thielscher A. Feasibility and resolution limits of opto-magnetic imaging of neural network activity in brain slices using color centers in diamond. Sci Rep. (2018) 8:4503. doi: 10.1038/s41598-018-22793-w

23. Sage DL, Arai K, Glenn DR, DeVience SJ, Pham LM, Rahn-Lee L, et al. Optical magnetic imaging of living cells. Nature. (2013) 496:486-9. doi: $10.1038 /$ nature 12072

24. Glenn DR, Lee K, Park H, Weissleder R, Yacoby A, Lukin MD, et al. Singlecell magnetic imaging using a quantum diamond microscope. Nat Methods. (2015) 12:736-8. doi: 10.1038/nmeth.3449

25. Davis HC, Ramesh P, Bhatnagar A, Lee-Gosselin A, Barry JF, Glenn $\mathrm{DR}$, et al. Mapping the microscale origins of magnetic resonance image contrast with subcellular diamond magnetometry. Nat Commun. (2018) 9:131. doi: 10.1038/s41467-017-02471-7

26. Kazi Z, Shelby I, Brunelle N, Watanabe H, Itoh KM, Wiggins P, et al. Widefield magnetic imaging of sub-50 $\mathrm{nm}$ ferromagnetic nanoparticles for timeresolved bio-mechanical orientation measurements. In: Conference on Lasers and Electro-Optics. San Jose, CA (2019). doi: 10.1364/cleo_at.2019.am4i.1

27. Glenn DR, Fu RR, Kehayias P, Sage DL, Lima EA, Weiss BP, et al. Micrometer-scale magnetic imaging of geological samples using a quantum diamond microscope. Geochem Geophys Geosyst. (2017) 18:3254-67. doi: 10.1002/2017gc006946

28. Schlussel Y, Lenz T, Rohner D, Bar-Haim Y, Bougas L, Groswasser $\mathrm{D}$, et al. Wide-field imaging of superconductor vortices with electron spins in diamond. Phys Rev Appl. (2018) 10:034032. doi: 10.1103/physrevapplied.10.034032

29. Lee M, Jang B, Yoon J, Mathpal MC, Lee Y, Kim C, et al. Magnetic imaging of a single ferromagnetic nanowire using diamond atomic sensors. Nanotechnology. (2018) 29:405502. doi: 10.1088/1361-6528/aad2fe

30. Jahnke KD, Naydenov B, Teraji T, Koizumi S, Umeda T, Isoya J, et al. Long coherence time of spin qubits in 12C enriched polycrystalline chemical vapor deposition diamond. Appl Phys Lett. (2012) 101:012405. doi: $10.1063 / 1.4731778$

31. Edmonds AM, Hart CA, Turner MJ, Colard PO, Schloss JM, Olsson K, et al. Generation of nitrogen-vacancy ensembles in diamond for quantum sensors: optimization and scalability of CVD processes. arXiv [Preprint]. arxiv: 2004.01746

32. Trusheim ME, Englund D. Wide-field strain imaging with preferentially aligned nitrogen-vacancy centers in polycrystalline diamond. New J Phys. (2016) 18:123023. doi: 10.1088/1367-2630/aa5040

33. El-Ella HAR, Ahmadi S, Wojciechowski AM, Huck A, Andersen UL. Optimised frequency modulation for continuous-wave optical magnetic resonance sensing using nitrogen-vacancy ensembles. Opt Express. (2017) 25:14809. doi: 10.1364/oe.25.014809

34. Bauch E, Hart CA, Schloss JM, Turner MJ, Barry JF, Kehayias P, et al. Ultralong dephasing times in solid-state spin ensembles via quantum control. Phys Rev X. (2018) 8:031025. doi: 10.1103/physrevx.8. 031025

35. Dréau A, Lesik M, Rondin L, Spinicelli P, Arcizet O, Roch JF, et al. Avoiding power broadening in optically detected magnetic resonance of single NV 
defects for enhanced dc magnetic field sensitivity. Phys Rev B. (2011) 84:195204. doi: 10.1103/physrevb.84.195204

36. Bougas L, Wilzewski A, Dumeige Y, Antypas D, Wu T, Wickenbrock A, et al. On the possibility of miniature diamond-based magnetometers using waveguide geometries. Micromachines. (2018) 9:276. doi: 10.3390/mi9060276

37. Sasaki K, Monnai Y, Saijo S, Fujita R, Watanabe H, Ishi-Hayase J, et al. Broadband, large-area microwave antenna for optically detected magnetic resonance of nitrogen-vacancy centers in diamond. Rev Sci Instrum. (2016) 87:053904. doi: 10.1063/1.4952418

38. Webb JL, Clement JD, Troise L, Ahmadi S, Johansen GJ, Huck A, et al. Nanotesla sensitivity magnetic field sensing using a compact diamond nitrogen-vacancy magnetometer. Appl Phys Lett. (2019) 114:231103. doi: 10.1063/1.5095241

39. Wojciechowski AM, Karadas M, Osterkamp C, Jankuhn S, Meijer J, Jelezko F, et al. Precision temperature sensing in the presence of magnetic field noise and vice-versa using nitrogen-vacancy centers in diamond. Appl Phys Lett. (2018) 113:013502. doi: 10.1063/1.5026678

40. Nöbauer T, Angerer A, Bartels B, Trupke M, Rotter S, Schmiedmayer J, et al. Smooth optimal quantum control for robust solid-state spin magnetometry. Phys Rev Lett. (2015) 115:190801. doi: 10.1103/physrevlett.115.190801

41. Chatzidrosos G, Wickenbrock A, Bougas L, Leefer N, Wu T, Jensen K, et al. Miniature cavity-enhanced diamond magnetometer. Phys Rev Appl. (2017) 8:044019. doi: 10.1103/physrevapplied.8.044019

42. Ahmadi S, El-Ella HAR, Wojciechowski AM, Gehring T, Hansen JOB, Huck A, et al. Nitrogen-vacancy ensemble magnetometry based on pump absorption. Phys Rev B. (2018) 97:024105. doi: 10.1103/physrevb.97.024105

43. Fescenko I, Jarmola A, Savukov I, Kehayias P, Smits J, Damron J, et al. Diamond magnetometer enhanced by ferrite flux concentrators. Phys Rev Res. (2020) 2:023394. doi: 10.1103/PhysRevResearch.2.023394

44. Jeske J, Cole JH, Greentree AD. Laser threshold magnetometry. New J Phys. (2016) 18:013015. doi: 10.1088/1367-2630/18/1/013015
45. Dumeige Y, Roch JF, Bretenaker F, Debuisschert T, Acosta V, Becher $\mathrm{C}$, et al. Infrared laser threshold magnetometry with a $\mathrm{NV}$ doped diamond intracavity etalon. Opt Express. (2019) 27:1706. doi: 10.1364/oe.27. 001706

46. Ibrahim MI, Foy C, Englund DR, Han R. 29.2 A scalable quantum magnetometer in $65 \mathrm{~nm}$ CMOS with vector-field detection capability. In: 2019 IEEE International Solid-State Circuits Conference (ISSCC) (San Francisco, CA: IEEE) (2019). doi: 10.1109/isscc.2019.8662434

47. Kim D, Ibrahim MI, Foy C, Trusheim ME, Han R, Englund DR. A CMOSintegrated quantum sensor based on nitrogen-vacancy centres. Nat Electron. (2019) 2:284-9. doi: 10.1038/s41928-019-0275-5

48. Stürner FM, Brenneis A, Kassel J, Wostradowski U, Rölver R, Fuchs $\mathrm{T}$, et al. Compact integrated magnetometer based on nitrogenvacancy centres in diamond. Diamond Relat Mater. (2019) 93:59-65. doi: 10.1016/j.diamond.2019.01.008

49. Huang TY, Grote RR, Mann SA, Hopper DA, Exarhos AL, Lopez GG, et al. A monolithic immersion metalens for imaging solid-state quantum emitters. Nat Commun. (2019) 10:2392. doi: 10.1038/s41467-019-10238-5

Conflict of Interest: The authors declare that the research was conducted in the absence of any commercial or financial relationships that could be construed as a potential conflict of interest.

Copyright (C) 2020 Webb, Troise, Hansen, Achard, Brinza, Staacke, Kieschnick, Meijer, Perrier, Berg-Sørensen, Huck and Andersen. This is an open-access article distributed under the terms of the Creative Commons Attribution License (CC BY). The use, distribution or reproduction in other forums is permitted, provided the original author(s) and the copyright owner(s) are credited and that the original publication in this journal is cited, in accordance with accepted academic practice. No use, distribution or reproduction is permitted which does not comply with these terms. 